\title{
Spatiotemporal Evolution of the Functional Magnetic Resonance Imaging Response to Ultrashort Stimuli
}

\author{
Yoshiyuki Hirano, Bojana Stefanovic, and Afonso C. Silva \\ Cerebral Microcirculation Unit, Laboratory of Functional and Molecular Imaging, National Institute of Neurological Disorders and Stroke, National \\ Institutes of Health, Bethesda, Maryland 20892-1065
}

The specificity of the hemodynamic response function (HRF) is determined spatially by the vascular architecture and temporally by the evolution of hemodynamic changes. The stimulus duration has additional influence on the spatiotemporal evolution of the HRF, as brief stimuli elicit responses that engage only the local vasculature, whereas long stimuli lead to the involvement of remote vascular supply and drainage. Here, we used functional magnetic resonance imaging to investigate the spatiotemporal evolution of the blood oxygenation level-dependent (BOLD), cerebral blood flow (CBF), and cerebral blood volume (CBV) HRF to ultrashort forelimb stimulation in an anesthetized rodent model. The HRFs to a single 333- $\mu$ s-long stimulus were robustly detected and consisted of a rapid response in both $\mathrm{CBF}$ and $\mathrm{CBV}$, with an onset time (OT) of $350 \mathrm{~ms}$ and a full width at half-maximum of $1 \mathrm{~s}$. In contrast, longer stimuli elicited a dispersive transit of oxygenated blood across the cortical microvasculature that significantly prolonged the evolution of the CBV HRF, but not the CBF. The CBF and CBV OTs suggest that vasoactive messengers are synthesized, released, and effective within 350 ms. However, the difference between the BOLD and CBV OT $(\sim 100 \mathrm{~ms})$ was significantly smaller than the arteriolar-venular transit time $(\sim 500 \mathrm{~ms})$, indicating an arterial contribution to the BOLD HRF. Finally, the rapid rate of growth of the active region with stimulus elongation suggests that functional hyperemia is an integrative process that involves the entire functional cortical depth. These findings offer a new view into the spatiotemporal dynamics of functional hemodynamic regulation in the brain.

\section{Introduction}

The most prominent human neuroimaging techniques, functional magnetic resonance imaging (fMRI), positron emission tomography, and diffuse optical imaging, map brain function by measuring the surrogate changes in cerebral blood flow (CBF), cerebral blood volume (CBV), and oxygenation, physiological quantities that accompany the changes in neural activity. Although a remarkable correspondence exists between changes in neural activity and the hemodynamic state, a complete understanding of the spatiotemporal evolution of the hemodynamic response function (HRF) is still missing.

Through neurovascular coupling, neurons and astrocytes release vasoactive agents (Iadecola and Nedergaard, 2007; Cauli and Hamel, 2010) that act on arterioles (Girouard and Iadecola, 2006) and capillaries (Chaigneau et al., 2003; Peppiatt et al., 2006; Stefanovic et al., 2008), causing local vasodilatation, decrease in vascular resistance, and increase in local blood volume and flow. The increased flow of blood through the capillary network leads

\footnotetext{
Received July 30, 2010; revised 0ct. 15, 2010; accepted Nov. 22, 2010.

This work was supported by the Intramural Research Program of National Institutes of Health-National Institute of Neurological Disorders and Stroke (Alan P. Koretsky, Scientific Director). We are deeply indebted to Dr. Alan P. Koretsky and Dr. Fernando F. Paiva for enlightening scientific discussions and to Xianfeng (Lisa) Zhang for her invaluable help in animal preparation and surgery.

Correspondence should be addressed to Dr. Afonso C. Silva, Cerebral Microcirculation Unit, National Institute of Neurological Disorders and Stroke, National Institutes of Health, 10 Center Drive, MSC 1065, Building 10, Room B1D106, Bethesda, MD 20892-1065. E-mail: silva@@ninds.nih.gov.

B. Stefanovic's present address: Imaging Research, Sunnybrook Health Sciences Centre, Toronto, 0N M4N 3M5, Canada.

DOI:10.1523/JNEUROSCI.3986-10.2011

Copyright $\odot 2011$ the authors $\quad 0270-6474 / 11 / 311440-08 \$ 15.00 / 0$
}

to inflow of oxyhemoglobin and decrease in deoxyhemoglobin in the venules and veins, the basis of the blood oxygenation leveldependent (BOLD) contrast (Ogawa et al., 1990). If the neuroglial activation is sustained, the dilatation propagates to the feeding arterioles and arteries (Iadecola et al., 1997), and increased blood oxygenation and vasodilatation spread to the draining venous vessels. Thus, the stimulus duration has a strong influence on the spatiotemporal characteristics of the HRF, as brief stimuli elicit responses that engage only the local vasculature, whereas longer stimuli lead to the involvement of remote vascular supply and drainage. Therefore, neuroimaging techniques sensitive to blood oxygenation, $\mathrm{CBF}$, or CBV measure signals that originate from different vascular compartments and have different temporal evolutions (Silva et al., 2007; Kennerley et al., 2010). A proper understanding of these distinct spatiotemporal features is crucial to the design and interpretation of functional brain-imaging experiments.

In the present work, we investigated the spatiotemporal characteristics of the BOLD, CBF, and CBV HRF to ultrashort focal brain stimulation in $\alpha$-chloralose-anesthetized rats. First, we studied the spatiotemporal dependence of the BOLD response on stimulus duration. Then, in a separate group of animals, we characterized the dynamic evolution of the BOLD, CBF, and CBV HRF across the cortical layers.

\section{Materials and Methods}

Animal preparation. Experiments were performed according to the guidelines of the National Institutes of Health Animal Care and Use Committee. Adult male Sprague Dawley rats $(n=18$; 205-349 g) were 
Table 1. Arterial blood gases and rectal temperature

\begin{tabular}{lccc}
\hline & BOLD $^{a}$ & BOLD/CBF & CBV \\
\hline $\mathrm{pH}$ & $7.36 \pm 0.05$ & $7.36 \pm 0.03$ & $7.39 \pm 0.03$ \\
$\mathrm{pCO}_{2}(\mathrm{mmHg})$ & $40.4 \pm 7.6$ & $36.7 \pm 4.0$ & $35.7 \pm 7.0$ \\
$\mathrm{pO}_{2}(\mathrm{mmHg})$ & $109 \pm 16$ & $103 \pm 14$ & $109 \pm 17$ \\
Rectal temperature $\left({ }^{\circ} \mathrm{C}\right)$ & $36.9 \pm 0.8$ & $37.1 \pm 0.5$ & $37.0 \pm 0.3$ \\
\hline
\end{tabular}

Arterial blood gases (mean $\pm S D$ ), corrected for actual rectal temperature, are shown.

${ }^{a}$ Arterial blood gases in BOLD-only experiments obtained from a separate group of animals $(n=8)$. BOLD/CBF and CBV experiments were both performed in the same set of animals $(n=9)$.

A

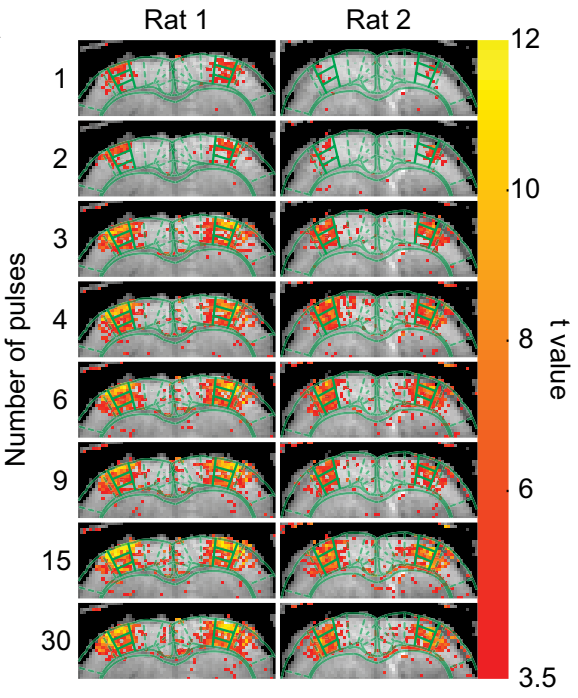

B
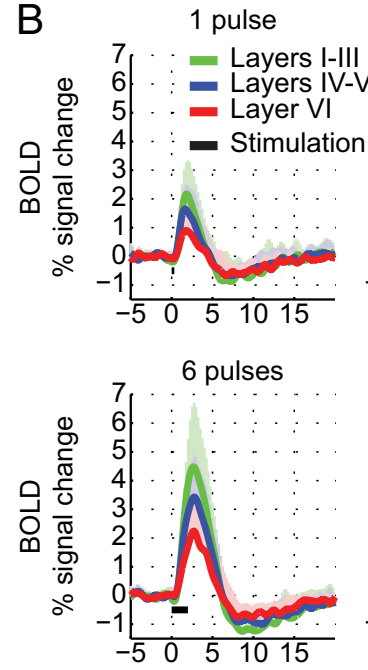

2 pulses
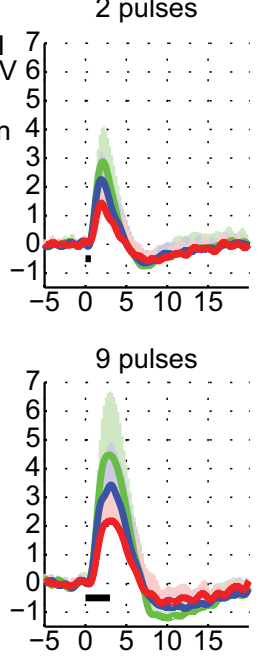

Time
C


$15 \mathrm{~cm}$ gradient set capable of $450 \mathrm{mT} / \mathrm{m}$ amplitude within $100 \mu \mathrm{s}$ rise time (Resonance Research). Radio frequency (RF) excitation was accomplished using a transmit-only volume RF coil and signal acquisition was performed via a receive-only surface coil positioned on top of the head. For arterial spin labeling (ASL) experiments, a butterfly-shaped labeling coil ( $1.7 \mathrm{~cm}$ inner diameter) was positioned under the neck of the animal, $\sim 2 \mathrm{~cm}$ away from isocenter (Silva et al., 1995).

fMRI was performed using a gradient-recall echo-planar imaging (EPI) sequence in a single coronal slice centered about bregma with the following parameters: field of view, $25.6 \times 12.8 \mathrm{~mm}^{2}$; nominal resolution, $200 \times 200 \times 2000 \mu^{3}$; echo time, $25 \mathrm{~ms}$; repetition time (TR), $250 \mathrm{~ms}$; flip angle, $30^{\circ}$. For simultaneous BOLD-CBF acquisition, a dynamic ASL (DASL) sequence (Barbier et al., 2001; Silva and Paiva, 2009) was used in which an ASL period of $199.7 \mathrm{~ms}$ was used and cycles of 120 label images and 120 control images were alternately acquired every $60 \mathrm{~s}$. For CBV measurements, $5 \mathrm{mg} / \mathrm{kg} 30 \mathrm{~nm}$ ultrasmall super-paramagnetic iron oxide particles (Molday ION; Biophysics Assay Laboratory, Worcester, MA) were injected intravenously to the animal after completion of the BOLD-CBF experiments. Slice-localized $B_{0}$ shimming was performed before all experiments.

Somatosensory stimulation. Bilateral electrical stimulation was performed via intradermal needle electrodes placed on both forelimbs (Silva et al., 2007). Stimulus delivery (333 $\mu$ s pulse width; $2 \mathrm{~mA}$ amplitude; $3 \mathrm{~Hz}$ pulse repetition) was controlled by a PC running Presentation (Neurobehavioral Systems). The stimulus paradigm consisted of 30-s-long epochs, in which a 10-slong prestimulus baseline period was followed by a period of bilateral forepaw stimulation of variable duration and by a poststimulus return-tobaseline period. Two sets of experiments were performed. First, in a group of eight animals, the stimulus duration was randomly varied among 1 , $2,3,4,6,9,15$, or 30 electrical pulses ( 30 pulses $\approx$ $10 \mathrm{~s}$ ) and repeated 10 times for a total run time of 40 min, during which the BOLD HRF was acquired. In a second set of experiments, performed in the remaining 10 animals, the stimulus duration was randomly varied among 1, 2, 3, 4, or 6 electrical pulses ( 6 pulses $\approx 2 \mathrm{~s}$ ) and repeated 16 times for measuring the BOLD and CBF HRF (run time, $80 \mathrm{~min}$ ), after which the CBV HRF was measured under the same paradigm (run time, $40 \mathrm{~min})$.

Data analysis. Data analysis was performed using custom software written in Matlab (The MathWorks). Data preprocessing consisted of in-plane motion correction using SPM5 (Statistical Parametric Mapping, Wellcome Trust Centre for Neuroimaging, University College London, London, UK). Baseline drift was removed and the series of 120 images (30 s) corresponding to each stimulus duration were identified and averaged across the epochs. High-frequency noise components were removed by filtering the averaged time courses durations of up to three pulses (1 s). B, Time courses of the BOLD HRF to stimuli of increasing durations, averaged across subjects $(n=8)$. C, Peak BOLD amplitude (top graph) and area under the curve (bottom graph) in each laminar ROI. In all layers, the peak BOLD amplitude increased with stimulus duration, reaching $90 \%$ of its maximum value at stimulus duration of six pulses ( $2 \mathrm{~s})$, after which it did not grow further. Error bars indicate 1SD.

anesthetized with isoflurane, mechanically ventilated, and catheterized. After surgery, anesthesia was switched to a continuous infusion of $\alpha$-chloralose (Silva and Koretsky, 2002). Rectal temperature, end-tidal $\mathrm{CO}_{2}$, respiratory tidal pressure, arterial blood pressure, and heart rate were continuously monitored. Arterial blood gases were sampled periodically and maintained at normal values throughout the experiments (Table 1).

Magnetic resonance imaging methods. fMRI experiments were performed in a $7 \mathrm{~T} / 30 \mathrm{~cm}$ AVI system (Bruker Biospin) and equipped with a with a $1 \mathrm{~Hz}$ square filter apodized with a $1.5 \mathrm{~Hz}$ Hanning window. One animal failed to respond to the stimuli and was excluded from the study.

After data preprocessing, region of interest (ROI) analysis was performed. First, the location of the forelimb area of primary somatosensory cortex (S1FL) regions was identified by warping the corresponding section of the rat stereotaxic atlas (Paxinos and Watson, 2007) to the EPI images (Keilholz et al., 2004). A functional $t$ map mask was generated by 
identifying regions of activation to the $6 \mathrm{~s}$ stimulus task. Then, the position of the S1FL ROI was further refined by coregistering its center of mass to the center of mass of the $t$ map mask. Once the final position of the S1FL ROI was determined, it was subdivided into three similarly sized regions according to the description of the cortical layers. The first sub-ROI included the pixels located along the top layers (layers I-III) of the cortex. The second subROI included pixels located along layers IV-V, and the third sub-ROI included pixels located along the bottom layer (layer VI) of the cortex. The number of pixels in each sub-ROI was $36.0 \pm 2.5$ pixels.

To quantify CBF, the DASL data were fitted to the tissue magnetization response model described by Equation 13.10 in the study by Silva and Paiva (2009). This modeling yields pixelby-pixel maps of resting CBF, the transit time between the labeling plane and the imaging slice, and the tissue T1 (Barbier et al., 2001; Silva and Paiva, 2009). Then, functional CBF values were determined by deconvolution of the DASL curve with the T1-decay curve (Silva et al., 2000). The BOLD, CBF, and CBV percentage signal changes were determined in each ROI. Onset time (OT), time to peak (TTP), full width at half-maximum (FWHM), peak amplitude, and area under the curve were calculated after a 16-fold Fourier interpolation. OT was defined as the minimal time after onset of stimulation necessary for the functional signals to consistently (for at least $1 \mathrm{~s}$ ) rise above 1 $\mathrm{SD}$ of the mean signal during the prestimulus period.

Data in graphs and tables are presented as mean \pm SD. Statistical analysis was performed using two-way ANOVA followed by Tukey's post hoc test. Unless specified otherwise, statistical significance was taken at $p<0.05$.

\section{Results}

\section{Saturation of BOLD amplitude with} stimulus duration

Figure 1 shows the results of the experiments to determine the spatiotemporal characteristics of the BOLD HRF to bilateral somatosensory stimulation as a function of stimulus duration. The region of activation (Fig. 1A) grew from just a few pixels located in the middle and upper layers of the S1FL in response to a single pulse stimulus, to occupy the entire S1FL areas and even include adjacent areas with longer stimulus durations of up to three pulses (1 s), beyond which no additional expansion was seen. Robust BOLD responses could be obtained from all three laminar regions for all stimulus durations (Fig. $1 B$ ). In agreement with previous results (Silva and Koretsky, 2002), layers I-III produced the strongest BOLD response, whereas layer VI produced the weakest BOLD response at any stimulus duration. The peak amplitude of the

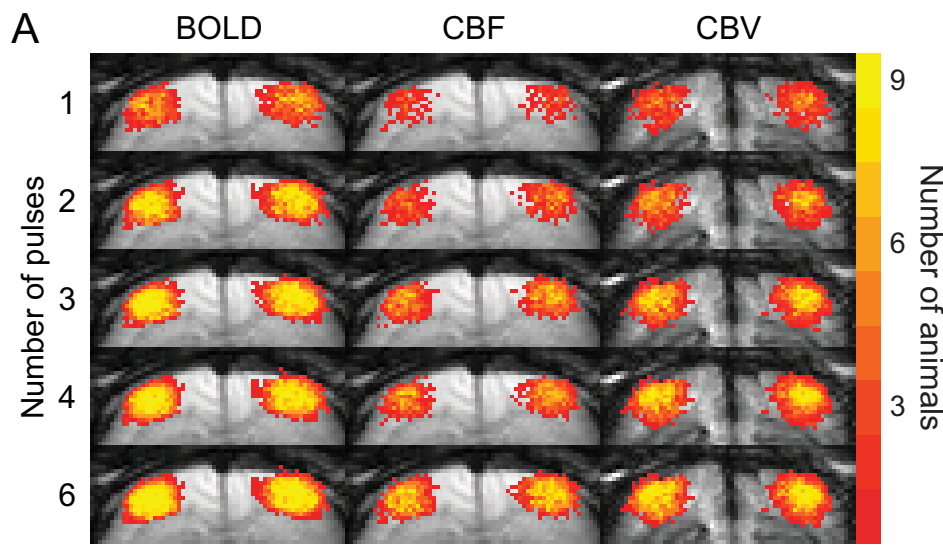

B



$\mathrm{CBF}$

CBV


Figure 2. $\quad \boldsymbol{A}$, Functional incidence maps for the BOLD, CBF, and CBV HRF to different stimulus durations $(n=9)$. Active pixels represent the locations that consistently (for at least $1 \mathrm{~s}$ ) clear 1 SD above the mean signal of the prestimulus period, colored according to the number of animals that show a response at that location. $B$, Laminar time courses of the BOLD, CBF, and CBV HRF to stimuli of increasing durations, averaged across subjects $(n=9)$. For all modalities, the peak amplitude was larger in layers I-III and IV-V than in layer VI. The CBF HRF showed both a quick onset and quick offset in response to all stimulus durations. The CBV HRF displayed a prolonged and slowly decaying offset tail, which was visible for stimulus durations as short as three pulses (arrows). Error bars indicate 1SD. 


\begin{tabular}{|c|c|c|c|c|c|}
\hline & \multicolumn{5}{|c|}{ Stimulus duration } \\
\hline & One pulse & Two pulses & Three pulses & Four pulses & Six pulses \\
\hline \multicolumn{6}{|c|}{ Layers I-III } \\
\hline BOLD & $1.0 \pm 0.5^{*}$ & $2.1 \pm 0.7^{*}$ & $2.9 \pm 1.1$ & $2.9 \pm 1.4$ & $3.4 \pm 1.6$ \\
\hline $\mathrm{CBF}$ & $32.5 \pm 21.8^{*}$ & $73.6 \pm 32.8^{*}$ & $93.4 \pm 37.8$ & $100.6 \pm 40.0$ & $107.5 \pm 41.4$ \\
\hline CBV & $2.9 \pm 1.2^{*}$ & $4.9 \pm 0.8^{*}$ & $6.7 \pm 2.0^{*}$ & $8.4 \pm 2.6^{*}$ & $10.4 \pm 3.3$ \\
\hline \multicolumn{6}{|c|}{ Layers IV-V } \\
\hline BOLD & $0.8 \pm 0.3^{*}$ & $1.9 \pm 0.5^{*}$ & $2.4 \pm 0.8^{*}$ & $2.6 \pm 0.9^{*}$ & $3.0 \pm 1.1$ \\
\hline $\mathrm{CBF}$ & $26.9 \pm 16.5^{*}$ & $67.0 \pm 30.0^{*}$ & $82.3 \pm 35.7^{*}$ & $97.5 \pm 43.1$ & $106.7 \pm 47.6$ \\
\hline CBV & $2.3 \pm 0.9^{*}$ & $4.4 \pm 1.2^{*}$ & $5.9 \pm 2.0^{*}$ & $6.9 \pm 2.3^{*}$ & $8.6 \pm 3.2$ \\
\hline \multicolumn{6}{|l|}{ Layer VI } \\
\hline BOLD & $0.5 \pm 0.2^{*}$ & $0.9 \pm 0.3^{*}$ & $1.2 \pm 0.4^{*}$ & $1.2 \pm 0.5$ & $1.4 \pm 0.5$ \\
\hline CBF & $22.9 \pm 14.3^{*}$ & $43.1 \pm 25.3^{*}$ & $50.4 \pm 19.3^{*}$ & $58.4 \pm 24.0$ & $60.6 \pm 28.4$ \\
\hline CBV & $0.9 \pm 0.4^{*}$ & $1.4 \pm 0.6^{*}$ & $1.9 \pm 0.9^{*}$ & $2.3 \pm 0.9$ & $2.7 \pm 1.2$ \\
\hline
\end{tabular}

*Significantly different from that of the six-pulse stimulation (paired $t$ test, $p<0.05$ ).

BOLD HRF grew from $\sim 2.3 \pm 1.0,1.7 \pm 0.9$, and $1.0 \pm 0.7 \%$ in layers I-III, IV-V, and VI, respectively, in response to a single pulse, to $4.6 \pm 2.2,3.5 \pm 1.4$, and $2.2 \pm 1.2 \%$, respectively, in response to six pulses $(2 \mathrm{~s})$. Longer stimulus durations produced no additional increases in the peak amplitude of the BOLD HRF (Fig. 1C). A small poststimulus undershoot signal could be detected in response to all stimulus durations, but its amplitude is not dependent on stimulus duration.

\section{Spatiotemporal characteristics of the BOLD, CBF, and CBV HRF to brief stimulation}

Based on the above observation that no additional increases in peak amplitude of the HRF occurred for stimulus durations beyond $2 \mathrm{~s}$, we set out to determine in additional detail the spatiotemporal characteristics of the BOLD, CBF, and CBV HRF to brief stimulation in a second group of animals. The functional incidence maps shown in Figure $2 A$ represent the robustness of detectability of the functional responses to each stimulus duration. CBF had the lowest detectability, whereas BOLD had the highest (supplemental Table S1, available at www.jneurosci.org as supplemental material). Although most rats showed robust BOLD response to all stimulus durations, not all rats presented a detectable CBF response because of the inherently low signal-tonoise ratio of ASL (Detre and Wang, 2002), which is further compromised under the conditions of short TR and thus low labeling efficiency of the DASL sequence used here (Silva and Paiva, 2009). For the dosage of iron oxide particles used (5 mg/ $\mathrm{kg}$ ), detectability of the CBV response was between that of the CBF and of the BOLD. For all three modalities, the highest incidence of activation happened in the central layers of the somatosensory cortex, with lower incidence in the pial surface and bottom layer and in the medial and lateral borders.

Both the amplitude and duration of the BOLD, CBF, and CBV HRFs (Fig. 2B) increased with longer stimulus durations. For all modalities, the peak amplitude was larger in layers I-III and IV-V than in layer VI of the cortex (Table 2$)(p<0.05)$. However, the peak amplitude of the CBF HRF saturated with stimulus durations of four pulses (Fig. 2B). In addition, the CBF HRF showed both a quick onset and quick offset in response to all stimulus durations. In contrast, while featuring a quick early onset, the CBV HRF displayed a prolonged offset tail (Silva et al., 2007), which was visible for stimulus durations as short as three pulses (1 s), becoming clearly evident in the HRF to four and six pulses (Fig. $2 B$, arrows).
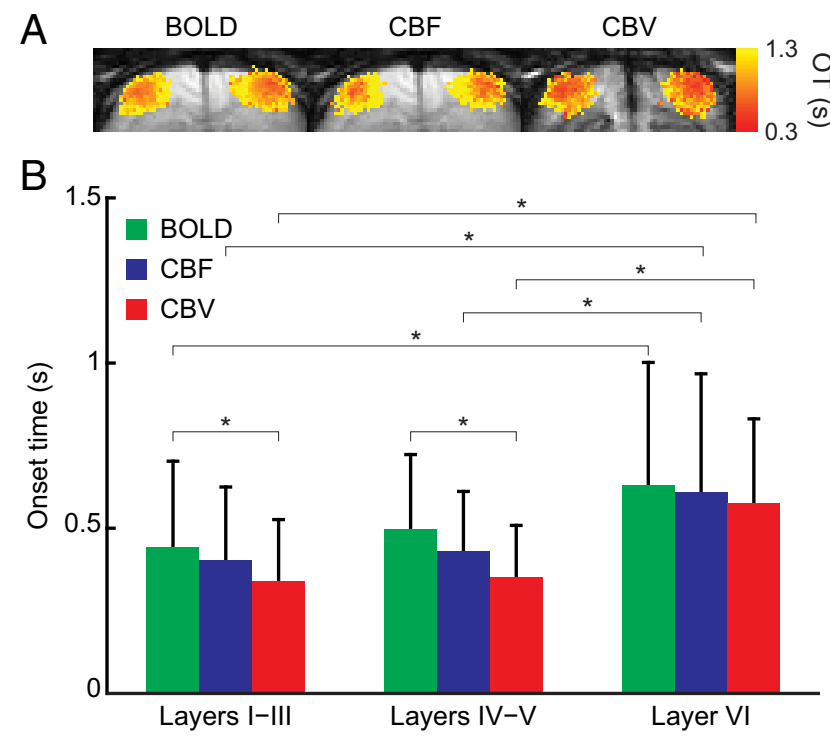

Figure 3. A, Mean onset time maps for the $B O L D, C B F$, and CBV HRF, averaged across subjects $(n=9)$ after spatial normalization to the rat brain atlas space (see Materials and Methods). The shortest onset times occurred in the middle cortical layers, whereas the longest onset times occurred in layer VI and in the adjacent cortical regions. $\boldsymbol{B}$, Graph of the mean onset times for the $B O L D, C B F$, and CBV HRF, averaged over all stimulus durations and across subjects $(n=9)$ as a function of the cortical depth. For all three functional modalities, the onset times in layer VI were consistently longer than in other layers. Across modalities, the CBV onset times in layers I-III and IV-V were significantly shorter than the respective BOLD onset times. Error bars indicate 1 SD. ${ }^{*} p<0.05$.

\section{CBF and CBV HRF have the shortest onset times}

Supplemental Table S2 (available at www.jneurosci.org as supplemental material) shows the OT, TTP, and FWHM of the HRFs for all stimulus durations. Figure $3 A$ shows the BOLD, CBF, and CBV mean OT maps averaged across subjects $(n=9)$ after spatial normalization to the rat brain atlas space (see Materials and Methods). Within each of the three functional modalities, the shortest OTs occurred in the middle and upper cortical layers, whereas the longest OTs occurred in layer VI and in the adjacent cortical regions. Because, by definition, OT is not dependent on stimulus duration, the OTs were averaged over the different stimulus durations for each of the three functional modalities and plotted in Figure $3 B$. Within each of the three functional modalities, the OTs in layer VI (BOLD, $0.63 \pm 0.33 \mathrm{~s}$; CBF, $0.61 \pm$ $0.34 \mathrm{~s}$; CBV, $0.58 \pm 0.25 \mathrm{~s}$ ) were consistently longer than in other layers $(p<0.001)$. However, no significant difference in OTs was observed between layers I-III and layers IV-V. Across the three functional modalities, whereas there was no difference in OTs in layer VI $(p>0.2)$, the CBV OTs in layers I-III $(0.34 \pm 0.19 \mathrm{~s})$ and layers IV-V $(0.35 \pm 0.16 \mathrm{~s})$ were significantly shorter than the respective BOLD OTs $(0.44 \pm 0.24$ and $0.50 \pm 0.21 s ; p<0.05)$, but not different from the respective CBF OTs $(0.40 \pm 0.22$ and $0.43 \pm 0.18 s ; p>0.15)$.

\section{Distinct temporal evolution of the CBV HRF}

After onset, the BOLD, CBF, and CBV HRF curves (Fig. 2B) evolved in amplitude to reach a peak response, followed by a return to baseline. As expected, both the TTP and FWHM in all laminar regions were directly correlated with the stimulus duration (supplemental Table S2, available at www.jneurosci.org as supplemental material). However, the dependence of TTP and FWHM on stimulus duration was different for each of the three 
functional modalities. The CBV TTP and FWHM were similar to the respective CBF parameters at short stimuli, but significantly longer than the $\mathrm{CBF}$ parameters for stimuli of long durations. This unique behavior of the CBV HRF can be better appreciated in Figure 4, which shows the BOLD, CBF, and CBV HRF time courses averaged across the nine rats and normalized to their respective peak amplitudes so that their dynamic characteristics can be easily compared. In all laminar regions, the onset dynamics of the CBV HRF to a single-pulse stimulus matches well the dynamics of the CBF HRF. Both modalities present similar TTP as well as FWHM $(\sim 1 s)$ (supplemental Table S2, available at www.jneurosci.org as supplemental material), $\sim 0.5$ s shorter than the corresponding BOLD parameters. As the stimulus duration increases, however, the CBV HRF presents a progressively longer TTP and FWHM that become similar to the corresponding BOLD parameters and significantly longer than the CBF TTP and FWHM, respectively.

TTP maps (Fig. 5A) show that, at all stimulus durations, the CBF has the shortest TTP, whereas BOLD has the longest. The CBV TTP at short stimuli durations is as short as the CBF TTP, but it elongates fast with increasing stimulus durations, becoming as long as the BOLD TTP at long stimulus durations. This differentiated behavior of the $\mathrm{CBV}$ dynamics can be better appreciated in Figure 5, $B$ and $C$, which shows the progression of TTP and FWHM versus stimulus duration for all three functional modalities. The graphs show that the CBF HRF has the shortest TTPs across all stimulus durations, whereas the BOLD has the longest. The CBV HRF presents TTP that is as short as the CBF TTP for short stimuli but as long as the BOLD TTP for long stimuli. The slopes of the linear regressions of TTP and FWHM for the CBV response (supplemental Table S3, available at www.jneurosci.org as supplemental material) were steeper than the corresponding slopes for the CBF response in all layers.

\section{Discussion}

We demonstrated that the hemodynamic response to a single 333- $\mu$ s-long stimulus consists of a rapid CBF and CBV evolution with onset times of $350 \mathrm{~ms}$ and full width at half-maxima of $1 \mathrm{~s}$. The BOLD, CBF, and CBV HRFs present distinct temporal behaviors across the cortical layers, which vary significantly with stimulus duration. In contrast to brief challenges, longer stimuli cause the appearance of a dispersive component that significantly prolongs the evolution of the CBV and the BOLD HRF, but not the CBF. Furthermore, we provided arguments for an arterial contribution to the BOLD HRF and to a significant contribution of venules and small intracortical veins to the CBV HRF with stimuli $>1$ s. Collectively, these findings show that functional hyperemia is an integrative process that is initiated within $350 \mathrm{~ms}$ of onset of neural activity and that involves all layers within the functional cortical depth, and offer a new view into the dynamics of functional hemodynamic regulation in the brain.
1 pulse
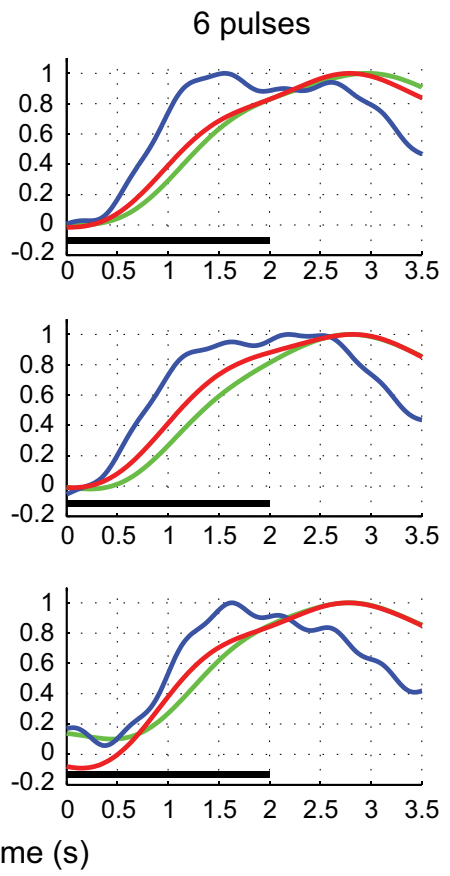

Time (s)

Figure 4. BOLD, CBF, and CBV HRF to stimulus durations of one pulse (left) or six pulses (right), averaged across subjects ( $n=$ and normalized to their respective peak amplitude. The temporal dynamics of the CBF and CBV HRF to a single-pulse stimulus were identical and faster than the evolution of the BOLD HRF in all layers. However, the CBV dynamics were as delayed as the BOLD dynamics in response to the $2 \mathrm{~s}$ stimulus, suggesting increased contribution of venules and veins to the CBV HRF to longer stimuli.

\section{Linearity of the BOLD HRF}

We determined that the peak amplitude of the BOLD HRF to somatosensory stimulation does not grow further for stimuli longer than six pulses ( $2 \mathrm{~s}$ ). Also, the CBF HRF presented saturation of its peak amplitude for stimuli longer than three pulses ( 1 s). The stimulus parameters used in the present work have been previously determined to be optimal in the $\alpha$-chloraloseanesthetized rat model of forepaw stimulation in eliciting the highest CBF (Silva et al., 1999) and BOLD (Gyngell et al., 1996; Keilholz et al., 2004; Huttunen et al., 2008; Sanganahalli et al., 2008) signal changes. The saturation of the BOLD amplitude at such short stimulus durations is consistent with a linear relationship between the BOLD response and the neural activity (Boynton et al., 1996; Pfeuffer et al., 2003; Birn and Bandettini, 2005) for stimulus durations up to four pulses (1.33 s) (Ogawa et al., 2000 ), in which both the amplitude and the area under the BOLD response increase linearly with stimulus duration. For stimulus durations longer than six pulses ( $2 \mathrm{~s}$ ), the BOLD peak amplitude becomes constant, whereas the area under the curve and FHWM increase linearly with stimulus duration (Pfeuffer et al., 2003). The BOLD HRF data shown in Figure 1 can be modeled by a constant stimulus function $S(t)$ of varying durations, multiplied by a neural modulation function (Boynton et al., 1996) of the form $N(t)=1.0+3.0 \cdot \exp (-t / 1.0)$ (Pfeuffer et al., 2003; Herman et al., 2009), convolved with a hemodynamic impulse response function given by a gamma variate with TTP of $1.5 \mathrm{~s}$ and FWHM of $1.6 \mathrm{~s}$. Although an analysis of the linearity of the HRF was not the purpose of the present study, the data are in agreement with previous studies that reported a linear, timeinvariant relationship between a small number of stimulation pulses applied to the rat forepaw and the area under the BOLD HRF curve (Ances et al., 2000; Ogawa et al., 2000; Herman et al., 2009). 
A

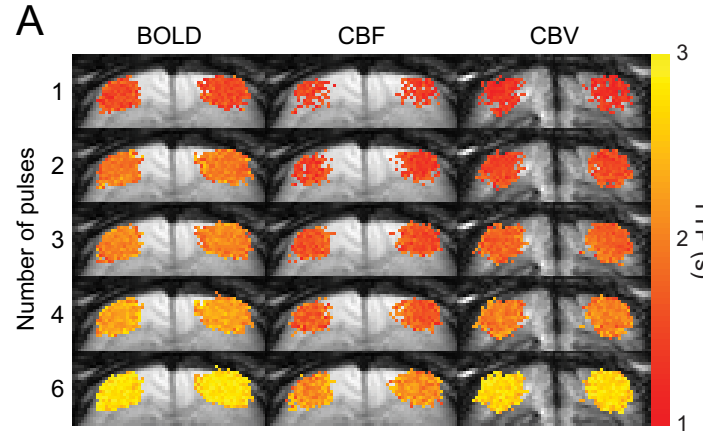

B

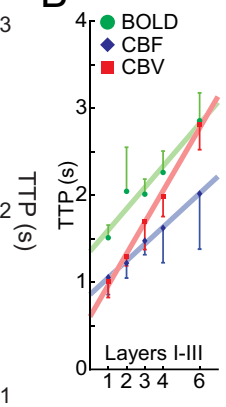



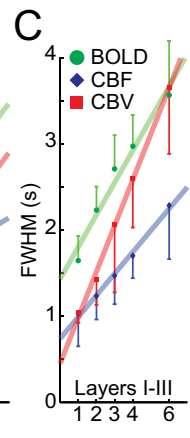

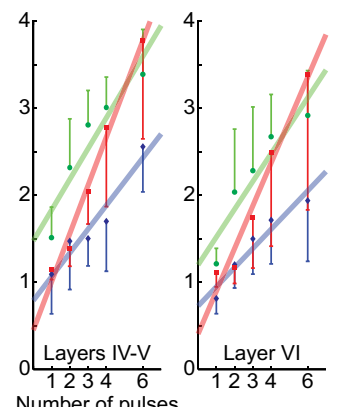

Figure 5. A, Mean TTP maps for the BOLD, (BF, and CBV HRF, averaged across subjects $(n=9)$ after spatial normalization to the rat brain atlas space (see Materials and Methods). At all stimulus durations, CBF has the shortest TTP, whereas BOLD has the longest. The CBV TTP with brief stimuli is as short as the CBF TTP, but it becomes as slow as the BOLD TTP with long stimuli. B, Plot of TTP versus stimulus duration in different cortical layers for all three functional modalities, averaged across subjects $(n=9)$. C, Plot of FWHM versus stimulus duration. Error bars indicate 1 SD.

\section{Comparison to previous work}

Although previous studies have reported on the fMRI response to brief stimulation of the rat forepaw (Ogawa et al., 2000; Herman et al., 2009; Sanganahalli et al., 2009), none of them focused on their spatial and temporal aspects. The highest incidence of activation happened in the central layers of the somatosensory cortex, in which the capillary density is highest (Woolsey et al., 1996; Weber et al., 2008), and by three pulses (1 s) stimulus duration, the entire S1FL region is active. This fast spatial filling of the entire S1FL shows that functional hyperemia, an integrative process, involves the entire functional cortical depth, in accordance with the fast spatial spreading of the neuronal activity across the cortical layers.

Compared with previous work performed at $11.7 \mathrm{~T}$ (Silva and Koretsky, 2002), the present data were acquired at $7 \mathrm{~T}$ using a single-shot echo-planar imaging sequence with lower contrastto-noise ratio and lower spatial and temporal resolution. Therefore, separation of the fMRI signals into the different cortical layers was much more difficult, and a smaller laminar difference in signal amplitude and OT between the upper and the middle cortical layers is reported here. Nevertheless, the present results are generally consistent with our previous work and bring in novel information on the CBF and CBV OT. To the best of our knowledge, the OTs reported here are the shortest measured to date and show that vasoactive agents are synthesized, released, and effective within $\sim 350 \mathrm{~ms}$ after neuronal activity. Interestingly, the OT difference between the BOLD and the CBF/CBV HRFs $(\sim 0.1 \mathrm{~s})$ is significantly smaller than the arteriole-venule transit time $(\sim 0.5 \mathrm{~s})$, suggesting that some fraction of the BOLD HRF originates on the arterial side of the vasculature. Supporting evidence for this inference comes from a recent report of measurable increases in oxygen saturation in small pial arteries on functional stimulation in the same animal model used here (Vazquez et al., 2010). This increase in arterial oxygenation, albeit small, would generate a BOLD signal on the arterial side with the same fast temporal characteristics observed here for the CBF and CBV $\mathrm{HRF}$, before the much larger increase in venous oxygenation that dominates BOLD contrast and slows down its temporal evolution. Our observation of a smaller difference in OT than in TTP between the BOLD and the $\mathrm{CBF} / \mathrm{CBV}$ is consistent with a small arterial contribution to the BOLD HRF.

\section{Estimation of arterial contribution to BOLD}

Under typical experimental conditions, the arterial contribution to the BOLD is expected to be quite small compared with the venous contribution. This is because the arterial contribution is limited by the small fractional increases in arterial oxygenation with functional increases in $\mathrm{CBF}$ as well as by the smaller relative effect of $\mathrm{PO}_{2}$ changes on $\mathrm{T}^{*}$ at high versus midrange basal $\mathrm{PO}_{2}$ values. Although proper estimation of the arterial contribution to BOLD would require development of a multicompartment biophysical model that takes into account mechanisms of oxygen transport along and across the vessels and $\mathrm{T} 2{ }^{*}$ dependence on vascular oxygenation, as well as parameters describing the physiological state of the blood, such as hematocrit, oxygen saturation levels in arteries and veins, and the hemoglobin oxygen dissociation curve, it is important to note that any arterial component of the BOLD signal is mostly relevant during the arteriole-venule transit time, before the arrival of oxygenated blood on the venous side of the vasculature, at which point the much stronger venous component starts dictating the amplitude and temporal characteristics of the BOLD curve. The amplitude of the BOLD response to a six-pulse stimulus, evaluated $0.5 \mathrm{~s}$ after stimulus onset, the arteriole-venule transit time (Hutchinson et al., 2006; Silva et al., 2007), is 5\% of its peak value (Fig. 4). Therefore, very simplistically, we estimate that the arterial contribution to the BOLD response to long stimuli under the present experimental conditions is $\sim 5 \%$. The relevance of this arterial BOLD component can be diminished, for example by increasing the arterial saturation of oxygen with hyperventilation or by increasing the oxygen content in the breathing air. Alternatively, the arterial contribution to BOLD can be further enhanced, for example by increasing the spatial resolution of the images to allow distinction of arterial versus venous components based on the spatial location and cortical arrangement of the different vessels, and/or by designing stimuli paradigms that attenuate the relative venous contribution to BOLD.

\section{Distinct temporal evolution of the BOLD, CBF, and CBV HRT}

The present data show that the BOLD, CBF, and CBV HRF have different temporal evolutions and distinct dependence on stimulus duration. For the briefest stimulus duration, TTP and FWHM for both the CBF and CBV HRF are virtually identical $(\sim 1 \mathrm{~s})$. As the stimulus duration increases, the temporal evolution of the CBV HRF becomes increasingly dissociated from that of the CBF HRF, presenting a biphasic behavior that has been well described previously (Mandeville et al., 1999; Silva et al., 2007). After stimulus offset, the CBV HRF decays initially rapidly but then slowly back to baseline, displaying a prolonged tail (Silva et al., 2007). Because the CBF HRF decays rapidly back to baseline, the slowly decaying tail of the CBV response most likely represents a passive vascular component of delayed compliance, which has been interpreted to be the slow relaxation of venules and veins 
during drainage of the increased amount of blood brought in by the functional HRF (Mandeville et al., 1999), a theory that inspired the balloon model (Buxton et al., 1998). Therefore, longer stimuli lead to a significant dispersive delay of the CBV HRF, and this should be considered when designing CBV-based experiments. Interestingly, the slow CBV dynamics at longer stimulus durations does not seem to compromise the spatial specificity of the CBV HRF (Zhao et al., 2005), as every active voxel in S1FL displays the biphasic response and the slowly decaying tail. This is attributable to two main reasons. First, below the pial surface, the cortical volume is rather uniformly filled by the microvasculature, with a regular distribution of large penetrating arteries and cortical veins that are interconnected by a dense capillary network (Weber et al., 2008). Second, the sensitivity of the iron oxide-based fMRI to CBV changes at the pial surface, where the large feeding arteries and draining veins are concentrated, has been shown to be small (Mandeville and Marota, 1999). These two factors combined suggest that the dispersive behavior of the CBV HRF has a significant contribution from venules and small intracortical veins (Kennerley et al., 2010).

\section{Implications to the dynamic regulation of functional hyperemia}

The spatiotemporal analysis of the present data provides significant and novel insight into the dynamic regulation of functional hyperemia. Functional brain stimulation elicits fast changes in $\mathrm{CBF}$ and $\mathrm{CBV}$, initiated mainly by an active redistribution of blood flow and volume within the capillary network (Hutchinson et al., 2006; Stefanovic et al., 2008) and local increases in blood flow and volume in the arterioles and venules. Precapillary increases in oxygenation and volume (Vazquez et al., 2010) translate into a small rapid arterial contribution to the BOLD HRF. The early increase in local blood flow is easily accommodated by the local microvasculature, so that the initial temporal evolution of CBF and CBV matches well, whereas the BOLD HRF lags $\sim 0.5$ $s$ behind because of influence of the arteriole-venule transit time (Hutchinson et al., 2006; Silva et al., 2007) on the BOLD signal. If the stimulus duration is short $(<1 \mathrm{~s})$ compared with the net arterial-venous transit time, $\mathrm{CBF}$ and CBV maintain a close temporal relationship, as the changes in blood flow and volume are equivalent both during the increased supply of blood on vasodilatation, as well as during the restoration of blood flow associated with vasoconstriction (Devor et al., 2007). Because of the contained involvement of the local microvasculature alone, functional experiments based on the use of very brief stimulus durations may be ideal in the study of the local, neuronally derived mediators of neurovascular coupling responsible for the regulation of the CBF response to fast, brief events (Cauli and Hamel, 2010).

As the stimulus duration increases, larger increases in $\mathrm{CBF}$ engage additional segments of the local vasculature, both on the arterial (Iadecola et al., 1997) and on the venous (Berwick et al., 2008) side. Arterioles and arteries dilate as vasodilatory signals propagate upstream of the region of activation (Iadecola et al., 1997), increasing local CBF and CBV. Meanwhile, the postcapillary side of the vasculature starts to "collect" the increased amount of blood, imposing a continued increase in local CBV. Under these conditions, the temporal evolution of the CBV response starts to be influenced by the passive accumulation and drainage of blood in venules and small intracortical veins, approaching the evolution of the BOLD HRF. fMRI experiments performed with intermediate stimulus durations may, therefore, provide interesting information as to the relative contribution of small arteries and venules to the functional HRF. In addition, these stimulus durations have the advantage of producing the highest signal amplitudes and minimal prolonged effects, allowing faster repetition of the stimulation epochs.

With the sustained stimulation, the arteries and arterioles will dilate to a steady-state value (Silva et al., 2007). At this point, the CBF HRF reaches a plateau and the CBV curve switches to a slower rate of increase, similar to that of the BOLD HRF. On cessation of the stimulus, the arterioles and arteries constrict, causing a rapid decrease in $\mathrm{CBF}$ and an equally rapid initial decrease in $\mathrm{CBV}$. Once the vessels have returned to their resting tone, the $\mathrm{CBF}$ response is back to baseline, whereas the $\mathrm{CBV}$ is influenced by the slow drainage of blood away from the activated region. Because of the ceased supply of oxygenated blood to the venous side of the vasculature, the BOLD response also returns to baseline and may display an undershoot if the CBV is still mostly elevated (Buxton et al., 1998; Mandeville et al., 1999). Experiments performed under these conditions of long stimulus durations produce high signal amplitudes, but have the disadvantage of taking long to reset to the original baseline, necessitating long interstimulus intervals and potentially involving interactions between the responses to adjacent epochs, which may complicate the interpretation of results.

\section{References}

Ances BM, Zarahn E, Greenberg JH, Detre JA (2000) Coupling of neural activation to blood flow in the somatosensory cortex of rats is timeintensity separable, but not linear. J Cereb Blood Flow Metab 20:921-930.

Barbier EL, Silva AC, Kim SG, Koretsky AP (2001) Perfusion imaging using dynamic arterial spin labeling (DASL). Magn Reson Med 45:1021-1029.

Berwick J, Johnston D, Jones M, Martindale J, Martin C, Kennerley AJ, Redgrave P, Mayhew JE (2008) Fine detail of neurovascular coupling revealed by spatiotemporal analysis of the hemodynamic response to single whisker stimulation in rat barrel cortex. J Neurophysiol 99:787-798.

Birn RM, Bandettini PA (2005) The effect of stimulus duty cycle and "off" duration on BOLD response linearity. Neuroimage 27:70-82.

Boynton GM, Engel SA, Glover GH, Heeger DJ (1996) Linear systems analysis of functional magnetic resonance imaging in human V1. J Neurosci 16:4207-4221.

Buxton RB, Wong EC, Frank LR (1998) Dynamics of blood flow and oxygenation changes during brain activation: the balloon model. Magn Reson Med 39:855-864.

Cauli B, Hamel E (2010) Revisiting the role of neurons in neurovascular coupling. Front Neuroenergetics 2:9.

Chaigneau E, Oheim M, Audinat E, Charpak S (2003) Two-photon imaging of capillary blood flow in olfactory bulb glomeruli. Proc Natl Acad Sci U S A 100:13081-13086.

Detre JA, Wang J (2002) Technical aspects and utility of fMRI using BOLD and ASL. Clin Neurophysiol 113:621-634.

Devor A, Tian P, Nishimura N, Teng IC, Hillman EM, Narayanan SN, Ulbert I, Boas DA, Kleinfeld D, Dale AM (2007) Suppressed neuronal activity and concurrent arteriolar vasoconstriction may explain negative blood oxygenation level-dependent signal. J Neurosci 27:4452-4459.

Girouard H, Iadecola C (2006) Neurovascular coupling in the normal brain and in hypertension, stroke, and Alzheimer disease. J Appl Physiol 100:328-335.

Gyngell ML, Bock C, Schmitz B, Hoehn-Berlage M, Hossmann KA (1996) Variation of functional MRI signal in response to frequency of somatosensory stimulation in alpha-chloralose anesthetized rats. Magn Reson Med 36:13-15.

Herman P, Sanganahalli BG, Blumenfeld H, Hyder F (2009) Cerebral oxygen demand for short-lived and steady-state events. J Neurochem 109 [Suppl 1]:73-79.

Hutchinson EB, Stefanovic B, Koretsky AP, Silva AC (2006) Spatial flowvolume dissociation of the cerebral microcirculatory response to mild hypercapnia. Neuroimage 32:520-530.

Huttunen JK, Gröhn O, Penttonen M (2008) Coupling between simultaneously recorded BOLD response and neuronal activity in the rat somatosensory cortex. Neuroimage 39:775-785. 
Iadecola C, Nedergaard M (2007) Glial regulation of the cerebral microvasculature. Nat Neurosci 10:1369-1376.

Iadecola C, Yang G, Ebner TJ, Chen G (1997) Local and propagated vascular responses evoked by focal synaptic activity in cerebellar cortex. J Neurophysiol 78:651-659.

Keilholz SD, Silva AC, Raman M, Merkle H, Koretsky AP (2004) Functional MRI of the rodent somatosensory pathway using multislice echo planar imaging. Magn Reson Med 52:89-99.

Kennerley AJ, Mayhew JE, Redgrave P, Berwick J (2010) Vascular origins of BOLD and CBV fMRI signals: statistical mapping and histological sections compared. Open Neuroimag J 4:1-8.

Mandeville JB, Marota JJ (1999) Vascular filters of functional MRI: spatial localization using BOLD and CBV contrast. Magn Reson Med 42:591-598.

Mandeville JB, Marota JJ, Ayata C, Zaharchuk G, Moskowitz MA, Rosen BR, Weisskoff RM (1999) Evidence of a cerebrovascular postarteriole windkessel with delayed compliance. J Cereb Blood Flow Metab 19:679-689.

Ogawa S, Lee TM, Kay AR, Tank DW (1990) Brain magnetic resonance imaging with contrast dependent on blood oxygenation. Proc Natl Acad Sci U S A 87:9868-9872.

Ogawa S, Lee TM, Stepnoski R, Chen W, Zhu XH, Ugurbil K (2000) An approach to probe some neural systems interaction by functional MRI at neural time scale down to milliseconds. Proc Natl Acad Sci U S A 97:11026-11031.

Paxinos G, Watson C (2007) The rat brain in stereotaxic coordinates, Ed 6. San Diego: Academic

Peppiatt CM, Howarth C, Mobbs P, Attwell D (2006) Bidirectional control of CNS capillary diameter by pericytes. Nature 443:700-704.

Pfeuffer J, McCullough JC, Van de Moortele PF, Ugurbil K, Hu X (2003) Spatial dependence of the nonlinear BOLD response at short stimulus duration. Neuroimage 18:990-1000.

Sanganahalli BG, Herman P, Hyder F (2008) Frequency-dependent tactile responses in rat brain measured by functional MRI. NMR Biomed 21:410-416.

Sanganahalli BG, Herman P, Blumenfeld H, Hyder F (2009) Oxidative neuroenergetics in event-related paradigms. J Neurosci 29:1707-1718.
Silva AC, Koretsky AP (2002) Laminar specificity of functional MRI onset times during somatosensory stimulation in rat. Proc Natl Acad Sci U S A 99:15182-15187.

Silva AC, Paiva FF (2009) Dynamic magnetic resonance imaging of cerebral blood flow using arterial spin labeling. Methods Mol Biol 489:277-295.

Silva AC, Zhang W, Williams DS, Koretsky AP (1995) Multi-slice MRI of rat brain perfusion during amphetamine stimulation using arterial spin labeling. Magn Reson Med 33:209-214.

Silva AC, Lee SP, Yang G, Iadecola C, Kim SG (1999) Simultaneous blood oxygenation level-dependent and cerebral blood flow functional magnetic resonance imaging during forepaw stimulation in the rat. J Cereb Blood Flow Metab 19:871-879.

Silva AC, Lee SP, Iadecola C, Kim SG (2000) Early temporal characteristics of cerebral blood flow and deoxyhemoglobin changes during somatosensory stimulation. J Cereb Blood Flow Metab 20:201-206.

Silva AC, Koretsky AP, Duyn JH (2007) Functional MRI impulse response for BOLD and CBV contrast in rat somatosensory cortex. Magn Reson Med 57:1110-1118.

Stefanovic B, Hutchinson E, Yakovleva V, Schram V, Russell JT, Belluscio L, Koretsky AP, Silva AC (2008) Functional reactivity of cerebral capillaries. J Cereb Blood Flow Metab 28:961-972.

Vazquez AL, Fukuda M, Tasker ML, Masamoto K, Kim SG (2010) Changes in cerebral arterial, tissue and venous oxygenation with evoked neural stimulation: implications for hemoglobin-based functional neuroimaging. J Cereb Blood Flow Metab 30:428-439.

Weber B, Keller AL, Reichold J, Logothetis NK (2008) The microvascular system of the striate and extrastriate visual cortex of the macaque. Cereb Cortex 18:2318-2330.

Woolsey TA, Rovainen CM, Cox SB, Henegar MH, Liang GE, Liu D, Moskalenko YE, Sui J, Wei L (1996) Neuronal units linked to microvascular modules in cerebral cortex: response elements for imaging the brain. Cereb Cortex 6:647-660.

Zhao F, Wang P, Hendrich K, Kim SG (2005) Spatial specificity of cerebral blood volume-weighted fMRI responses at columnar resolution. Neuroimage 27:416-424. 